\title{
The association of the level of self-care on adherence to treatment in patients diagnosed with type 2 diabetes
}

\author{
Sylwia Krzemińska ${ }^{1}$ (D) Katarzyna Lomper ${ }^{1}$ (D) $\cdot$ Anna Chudiak $^{1}$ (D) $\cdot$ Davide Ausili $^{2}$ (D) $\cdot$ Izabella Uchmanowicz $^{1}$ (D)
}

Received: 23 July 2020 / Accepted: 27 October 2020 / Published online: 29 November 2020

(c) The Author(s) 2020

\begin{abstract}
Aims The study aimed to assess the impact of self-care on adherence to treatment in patients diagnosed with type 2 diabetes and effect of complex interaction of social, lifestyle, economic, environmental and behavioural.

Methods The study was carried out between June 2018 and May 2019 on 324 patients (162 females, 162 males) with type 2 diabetes. To measure the levels of self-care, the Self-Care of Diabetes Index (SCODI) questionnaire was used. Adherence to treatment was assessed with the Adherence in Chronic Diseases Scale (ACDS).

Results The highest scores of health behaviour were on the subscale of adherence with the mean value of 68.37 , and the lowest results on the subscale of blood sugar self-monitoring, with the mean of 56.05. We found that low adherence to treatment was present in $52.47 \%$ of respondents, the moderate level in $39.20 \%$, while only $8.33 \%$ of patients showed the high level. There were significant positive correlations between the ACDS and SCODI subscales $(p<0.05)$ : self-care maintenance (0.436), self-care management (0.413), self-care monitoring (0.384), and self-care confidence (0.453).

Conclusions Self-care affects on adherence in patients with type 2 diabetes. The higher self-efficacy in each of the areas of functioning, the higher the level of adherence to treatment. We found that demographic variables such as female sex, education and employment status can influence self-care in managing chronic illnesses such as type 2 diabetes.
\end{abstract}

Keywords Nursing · Type 2 diabetes $\cdot$ Self-care $\cdot$ Adherence to treatment $\cdot$ Self-care of diabetes index (SCODI) Adherence in chronic diseases scale (ACDS)

This article belongs to the topical collection Health Education and Psycho-Social Aspects, managed by Massimo Porta and Marina Trento.

Izabella Uchmanowicz

izabella.uchmanowicz@umed.wroc.pl

Sylwia Krzemińska

sylwia.krzeminska@umed.wroc.pl

Katarzyna Lomper

katarzyna.lomper@umed.wroc.pl

Anna Chudiak

anna.chudiak@umed.wroc.pl

Davide Ausili

davide.ausili@unimib.it

1 Department of Clinical Nursing, Faculty of Health Sciences, Wroclaw Medical University, Wroclaw, Poland

2 Department of Medicine and Surgery, University of Milano-Bicocca, Monza, Italy

\section{Introduction}

The epidemiological data show a significant worldwide growth of people with diabetes melitus (DM), which is currently one of the leading causes of global mortality [1]. In addition to genetic predispositions, it is important to stress the importance of the so-called modifiable factors, such as a sedentary lifestyle and unhealthy diet, which are significant predictors of the growing diabetes epidemic [2]. The disease has grown into a major public healthcare problem. The research on the subject reveals that in 2017 there were 425 million people with DM, and this number will more likely rise to 693 million by 2045 [3].

Uncontrolled (out of the therapeutic range) DM and chronic hyperglycaemia are a cause of severe health conditions mainly associated with damaging the vascular system but may also impair eyesight, nerves, kidneys and the heart [4]. Regular check-ups and follow up of patients diagnosed with DM may help prevent or delay health complications. Long-term measures present a great challenge to healthcare 
workers, as the needs of patients as regards self-care include not only regular blood glucose testing but also measures aimed at preventing the complications of the disease, including disability. Self-monitoring, which is referred to in the literature as diabetes-related self-care activities (DRSCAs), is a key element in the management of DM. DRSCAs relate to the active and effective self-management of the condition by the patient. Self-monitoring is a strategy focusing primarily on self-organisation of patient's life to ensure adherence to medication but also to monitor meals, blood glucose monitoring, physical activity and the practice of self-care routines, e.g. feet care [5].

American Diabetes Association (ADA) [6] stresses the importance of health education and self-care as a key element in the management of diabetic patients. Health education is of particular importance, as patients with type $2 \mathrm{DM}$ face numerous challenges like regular check-ups, adherence to treatment and self-care. An adequate health education is associated with good metabolic control and helps to prevent of compliactions associated with DM [7]. The main goal of health education among patients with DM is to achieve changes in health behaviours [8]. However, patients often find self-management difficult to engage in the treatment process on a daily basis, especially regarding daily blood glucose monitoring. As many as $85.7 \%$ of DM patients fail to achieve therapeutic haemoglobin A1C (HbA1c) levels [6].

The abnormal blood glucose levels in DM patient population may result from wrong nutrition habits, poor physical activity and non-adherence to treatment [9]. The available literature indicates many factors influencing the phenomenon of non-adherence in patients with diagnosed DM. Several studies carried out to date have discovered that education and economic status are key determinants of adherence in the population discussed. For instance, patients who are unemployed, younger and those with tertiary education are more likely to adhere to treatment [10]. The additional factors that may have a negative influence on blood glucose levels in DM patients also include insufficient knowledge, low self-esteem and none or poor social and family support. Recent study proves that a patient's family is also important in supporting patient's self-management behaviours [11]. Obviously, the literature reports other multiple factors which have an influence on adherence to treatment and the level of self-care in diabetes patients. For instance, promoting health behaviours by clinicians and healthcare workers is out of questioning.

Recent research findings on patients with chronic conditions suggest to take multifaceted efforts in order to improve adherence and self-care in this group of patients [12]. DM self-care involves not only daily blood glucose testing and adherence to medication but also modification of lifestyle, including diet, as well as a number of other measures, which should be supported by medical personnel. Indeed, effective management of DM is a difficult task for patients who very often do not understand the bottom line of self-care [13].

The aim of this study was to assess the impact of self-care on adherence to treatment in patients diagnosed with type $2 \mathrm{DM}$ and effect of complex interaction of social, lifestyle, economic, environmental and behavioural.

\section{Methods}

\section{Design and participants}

This observational and correlational study was carried out between June 2018 and May 2019 at the Internal Medicine Department of Hospital in Trzebnica (Poland), and the Diabetes Unit of Outpatient Clinic in Wroclaw (Poland). The STROBE guidelines (Strengthening the Reporting of Observational Studies in Epidemiology) were followed.

The study included 180 patients with diagnosed type 2 diabetes from each centre (360 patients in total). Participants included in the study met the following criteria for inclusion: type $2 \mathrm{DM}$, over 18 years of age, duration of illness of over 1 year, lack of cognitive disorders, ability to complete questionnaires on one's own, and patient's consent to take part in the study.

Due to incompleteness of research questionnaires or resignation from participation in the study finally, a group of 324 patients (162 females, 162 males) with type 2 DM was involved into analysis. Duration of illness was as follows: up to 5 years for 123 patients (37.96\%), 5-10 years for 80 (24.69\%), over 10 years for $120(37.04 \%)$, and no data available in case of $0.31 \%$ patients.

\section{Research tools}

In order to assess the level of self-care, the Self-Care of Diabetes Index (SCODI) questionnaire was used to measure self-care in DM patients. The questionnaire assesses the selfefficacy of a diabetes patient in four areas of daily living: self-care maintenance, self-care monitoring, self-care management and self-care confidence. Respondents' scores range from 0 to 100 , with the higher score indicating the higher level of self-efficacy in each domain of self-care behaviours. However, there are no available standards as to what scores indicate a high level of self-efficacy and what scores indicate a low level of self-efficacy. As all the areas are scored on the same scale, it is possible to compare the level of self-efficacy in different areas in order to identify any self-care deficits [14]. The study used the Polish adaptation of the tool. The SCODI questionnaire has acceptable internal consistency and reliability in assessing self-care among diabetic patients in the Polish population with Cronbach's Alpha: self-care 
maintenance (0.759), self-care monitoring (0.741), self-care management (0.695) and self-care confidence (0.932) [15].

The level of adherence to treatment was assessed with the Adherence in Chronic Diseases Scale (ACDS), which is designed for the assessment of patients with chronic conditions. The questionnaire contains 7 questions. Questions 1-5 address behaviours directly influencing adherence to treatment, whereas questions 6-7 examine/test patients' convictions and situations which have an indirect influence on adherence. The possible score range is 0 to 28 - the higher the score, the higher the level of adherence to treatment [16].

\section{Ethical considerations}

The study was approved by the Wroclaw Medical University Bioethics Committee (approval no. KB-622/2018). All patients provided informed consent, and were informed that they could withdraw from the study at any time. The study was carried out in accordance with the tenets of the Declaration of Helsinki and Good Clinical Practice guidelines.

\section{Statistical analyses}

A multiple factor analysis of the effect of independent variables was carried out using linear regression. The results were presented as the values of the regression model parameters with a $95 \%$ confidence interval. A significance level of 0.05 was used in the analysis. Thus, all $p$ values of less than 0.05 were interpreted as indicating a strong correlation. To assess how well the model fits the data, we used the R-squared determination coefficient. This measure is the proportion of the variance in the depedent variable that is explained by the model. The analysis was carried out using the R software, version 3.6.0 [17].

\section{Results}

\section{Sociodemographic and clinical analysis}

The study was performed on 324 patients (162 females, 162 males). Over half of the respondents were aged over 60 $(54.01 \%)$. The largest group of respondents were those who had been diagnosed up to 5 years before (37.96\%), followed by those who had been diagnosed over 10 years before the study $(37.04 \%)$. As regards the place of residence, $68.83 \%$ of the respondents lived in urban areas and $30.56 \%$ lived in rural areas. As for the level of education, $34.57 \%$ of the respondents had secondary education, $34.26 \%$ vocational education, $17.9 \%$ tertiary education and $13.27 \%$ primary education. Of the respondents, $37.96 \%$ were retired, $37.35 \%$ declared that they were employed, $13.58 \%$ were retired on ill-health grounds and $11.11 \%$ were unemployed. The data are shown in Table 1.
Table 1 Sociodemographic and clinical analysis $(N=324)$

\begin{tabular}{lll}
\hline Feature & & Values (\%) \\
\hline Sex & Female & $162(50.00)$ \\
Age & Male & $162(50.00)$ \\
& Up to 60 years & $149(45.99)$ \\
Place of residence & Over 60 years & $175(54.01)$ \\
& Urban area & $223(68.83)$ \\
& Rural area & $99(30.56)$ \\
Education & No data available & $2(0.62)$ \\
& Primary & $43(13.27)$ \\
& Vocational & $111(34.26)$ \\
Source of income & Secondary & $112(34.57)$ \\
& Tertiary & $58(17.90)$ \\
& Employed & $121(37.35)$ \\
& Unemployed & $36(11.11)$ \\
& Retired & $123(37.96)$ \\
Duration of diabetes & Retired on ill-health grounds & $44(13.58)$ \\
& Up to 5 years & $123(37.96)$ \\
& 5-10 years & $80(24.69)$ \\
& Over 10 years & $120(37.04)$ \\
& No data available & $(0.31)$ \\
\hline
\end{tabular}

\section{Assessment of the level of self-care with SCODI questionnaire}

The results of the SCODI questionnaire showed that the respondents scored the highest on adherence to recommendations concerning self-care maintenance, with an average of 68.37 points $(\mathrm{SD}=18.14, \mathrm{Me}=68.75)$, and the lowest on self-care monitoring, with an average of 56.05 points $(\mathrm{SD}=22.45, \mathrm{Me}=55.56)$. The data are shown in Table 2 .

\section{Assessment of adherence to treatment using the ACDS questionnaire}

The assessment of adherence to treatment showed that $52.47 \%$ of the respondents presented a low level of adherence, $39.20 \%$ - a moderate level of adherence and only $8.33 \%$ - a high level of adherence. The data are shown in Table 3.

\section{Correlation between adherence to treatment and the level of self-care}

There was a significant positive correlation between the ACDS questionnaire scores and each subscale of the SCODI questionnaire $(p<0.05)$. Therefore, the higher the level of adherence to treatment, the higher the level of 
Table 2 Results of the SCODI questionnaire

\begin{tabular}{lllllllll}
\hline SCODI & $\mathrm{N}$ & $\mathrm{M}$ & $\mathrm{SD}$ & $\mathrm{Me}$ & $\mathrm{Min}$ & $\mathrm{Max}$ & $\mathrm{Q} 1$ & $\mathrm{Q} 3$ \\
\hline Self-care maintenance & 324 & 68.37 & 18.14 & 68.75 & 12.5 & 139.58 & 56.25 & 81.25 \\
Self-care management & 324 & 63.85 & 24.34 & 64.71 & 0 & 211.76 & 50 & 82.35 \\
Self-care monitoring & 324 & 56.05 & 22.45 & 55,56 & 0 & 240.62 & 43.75 & 67.19 \\
Self-care confidence & 324 & 64.45 & 20.38 & 63.64 & 4.55 & 100 & 52.27 & 79.55 \\
\hline
\end{tabular}

$S C O D I$ Self-Care of Diabetes Index, $N$ number of patients, $M$ mean, $S D$ standard deviation, $M e$ median, Min minimum value, Max maximum value, $Q 1$ quartile 1st, $Q 3$ quartile 3 rd
Table 3 Results of the ACDS questionnaire

\begin{tabular}{llll}
\hline ACDS_-score & Interpretation & $N$ & $\%$ \\
\hline $0-20$ & Low level of adherence & 170 & $52.47 \%$ \\
$21-26$ & Moderate level of adherence & 127 & $39.20 \%$ \\
$27-28$ & High level of adherence & 27 & $8.33 \%$ \\
\hline
\end{tabular}

ACDS Adherence in Chronic Diseases Scale, $N$ number of patients

self-efficacy in each of the areas. The data are shown in Table 4 and Fig. 1.

\section{Multiple factor analyses of the SCODI questionnaire}

"Self-care maintenance" subscale. The linear regression model showed that the independent predictors of self-care maintenance were the following variables $(p<0.05)$ : (1) ACDS score/adherence outcome/adherence ability: each additional point scored in the ACDS questionnaire increased the subscale score by an average of 1.476 points; (2) Sex: compared with female respondents, the subscale score for male respondents was lower by an average of 8.869 points; (3) Education: compared with respondents with primary education, the score for respondents with tertiary education was higher by an average of 9.241 points. The $\mathrm{R}^{2}$ coefficient yielded the level of $31.23 \%$. The data are shown in Table 5a.

"Self-care management" subscale. The linear regression model showed that the independent predictors of behaviours such as self-care management behaviours were the following variables $(p<0.05)$ : $(1)$ adherence factor (ACDS score): each additional point scored in the ACDS questionnaire increased the subscale score by an average of 1.797 points; (2) Sex: compared with female respondents, the subscale score for male respondents was lower by an average of 5.3 points; (3) Education: compared with respondents with primary education, the subscale score for respondents with vocational education was higher by an average of 10.716 points, the score for the respondents who had secondary education was higher by an average of 10.399 points and the score for respondents with tertiary education was higher by an average of 16.554 points; (4) Source of income: compared with the respondents who were employed, the subscale score for unemployed respondents was higher by an average of 11.865 points, and the score for the respondents who were retired on illhealth grounds was lower by an average of 8.844 points. The $\mathrm{R}^{2}$ coefficient was at the level of $29.84 \%$. The data are shown in Table 5a.

"Self-Care monitoring" subscale. The linear regression model showed that the independent predictors of a score on Self-care monitoring were the following variables $(p<0.05)$ : (1) adherence outcome/adherence ability: each additional point scored in the ACDS questionnaire increased the subscale score by an average of 1.59 points; (2) Education: compared with the respondents who had primary education, the score for respondents with secondary education was higher by an average of 11.02 points, and the score for respondents with tertiary education was
Table 4 Correlation between the level of adherence and selfcare ability

\begin{tabular}{lllll}
\hline SCODI & \multicolumn{2}{l}{ Correlation with the ACDS } & \\
\cline { 2 - 5 } & $\begin{array}{l}\text { Correlation coef- } \\
\text { ficient }\end{array}$ & $p^{*}$ & $\begin{array}{l}\text { Direction of cor- } \\
\text { relation }\end{array}$ & $\begin{array}{l}\text { Strength of } \\
\text { correlation }\end{array}$ \\
\hline Self-care maintenance & 0.436 & $p<0.001 \mathrm{NP}$ & Positive & Weak \\
Self-care management & 0.413 & $p<0.001 \mathrm{NP}$ & Positive & Weak \\
Self-care monitoring & 0.384 & $p<0.001 \mathrm{NP}$ & Positive & Weak \\
Self-care confidence & 0.453 & $p<0.001 \mathrm{NP}$ & Positive & Weak \\
\hline
\end{tabular}

${ }^{*} P=$ Normal (parametric) distribution of both the correlated variables, Pearson correlation coefficient; $\mathrm{NP}=$ Non-parametric distribution (lack of normal distribution) in the case of at least one of the correlated variables, Spearman's correlation coefficient

SCODI Self-Care of Diabetes Index, ACDS Adherence in Chronic Diseases Scale, $N$ number of patients, $p$ level of statistical significance 

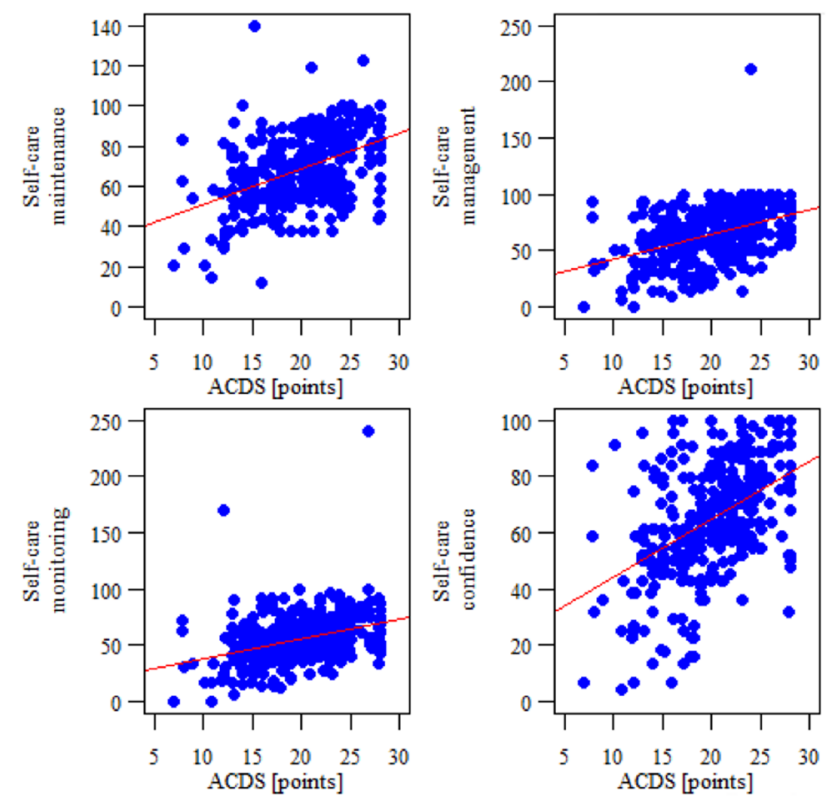

Fig. 1 Correlation between the level of adherence and self-care ability

higher by an average of 14.347 points. The $\mathrm{R}^{2}$ coefficient was at the level of $20.4 \%$. The data are shown in Table $5 \mathrm{~b}$.

"Self-care confidence" subscale. The linear regression model showed that the independent predictors of self-care confidence were the following $(p<0.05)$ : (1) ACDS score adherence outcome/adherence ability: each additional point scored in the ACDS questionnaire increased the subscale score by an average of 1.834 points; (2) Education: compared with the respondents who had primary education, the score for respondents with vocational education was higher by an average of 7.64 points, the score for respondents with secondary education was higher by an average of 9.962 points and the score for respondents with tertiary education was higher by an average of 16.901 points; (3) Source of income: compared with the respondents who were employed, the subscale score for unemployed respondents was higher by an average of 7.902 points. The $\mathrm{R}^{2}$ coefficient was at the level of $31.67 \%$. The data are shown in Table $5 b$.

The $\mathrm{R}^{2}$ coefficient, i.e. the proportion of the variance of the results which is explained by the variables included in a given model, for the above models ranged from $20.40 \%$ (for selfcare managemen) to $31.67 \%$ (for self-care confidence). Thus, the proportion of the variance which is explained by variables not included in the models and random factors ranged from $68.33 \%$ to $79.60 \%$.

\section{Discussion}

DM is one of the fastest growing diseases in the world. Despite the availability of treatment, patients do not manage to control the disease due to failure to comply with therapeutic, dietary and physical activity guidelines. Contemporary health care practice is to promote and support people with chronic illness to self-care management [18]. For that reasons, quantitative assessment how self-care affects adherence to treatment in patients diagnosed with type $2 \mathrm{DM}$ becomes a very important for nursing practice. Identifying causes for poor or none of self-care behaviours, and non-adherence in DM patients, requires that relevant steps be taken to enhance the self-efficacy of patients and improve their treatment outcomes.

The existing studies suggest that patients diagnosed with DM have a moderate level of knowledge of their condition and that their level of knowledge depends mainly on such factors as age, education and duration of illness. It is necessary to provide patients with health education tailored to their needs. Healthcare workers play a major role in identifying problems and prepare suitable programmes on that basis [19]. The latest clinical recommendations of the Polish Diabetes Association show that blood glucose monitoring and analysis is an integral part of the management of DM. The document stresses how important is the systematic education of patients about self-monitoring, including education information about a glucose metre and ways of interpreting measurements regarding changes of diet, physical activity and medication doses [20]. We ran the study on a group of 324 patients diagnosed with type 2 DM. The patients scored the highest on adherence to self-care maintenance recommendations and the lowest on self-care monitoring. The literature mentions seven basic behaviours relating to diabetes self-care which are essential to good treatment outcomes. They include a healthy diet, physical activity, blood glucose monitoring, adherence to medication, ability to solve problems, ability to cope with stress as well as risk mitigating behaviours [21]. These behaviours were found to correlate positively with good blood glucose levels, reduction of complications, improved quality of life and improved adherence to treatment [22-24].

Adherence to treatment in chronic conditions is a significant issue raised in the recent literature. Our study found that $52.47 \%$ of the respondents showed the low level of adherence, $39.20 \%$ of patients adhered at the moderate level and only $8.33 \%$ of patients presented the high adherence to treatment, which confirms how serious the problem is Poland in terms of self-management activities. A similar study by Polonsky and Henry [25] carried out on patients with DM found that only $30 \%$ of study participants adhered to treatment regimens and that patients with lower socio-economic 
Table 5 Multiple factor analysis of the "Self-care maintenance", "Self-care management", "Self-care monitoring" and "Self-care confidence" subscales of the SCODI questionnaire

\begin{tabular}{|c|c|c|c|c|c|c|}
\hline \multirow[t]{2}{*}{ Variable } & $R$ & $95 \% \mathrm{CI}$ & $p$ & $R$ & $95 \%$ CI & $p$ \\
\hline & \multicolumn{3}{|c|}{ "Self-care maintenance" } & \multicolumn{3}{|c|}{ "Self-care management" } \\
\hline
\end{tabular}

\begin{tabular}{|c|c|c|c|c|c|c|c|c|c|}
\hline & & & \multicolumn{7}{|c|}{ (a) } \\
\hline & ACDS [points] & 1.476 & 1.088 & 1.864 & $<0.001$ & 1.797 & 1.271 & 2.324 & $<0.001$ \\
\hline \multirow[t]{2}{*}{ Sex } & Female & ref & & & & ref & & & \\
\hline & Male & -8.869 & -12.457 & -5.28 & $<0.001$ & -5.3 & -10.176 & -0.424 & 0.033 \\
\hline \multirow[t]{2}{*}{ Age } & Up to 60 years & ref & & & & ref & & & \\
\hline & Over 60 years & -0.547 & -6.024 & 4.931 & 0.844 & -6.452 & -13.894 & 0.991 & 0.089 \\
\hline \multirow[t]{2}{*}{ Place of residence } & Urban area & ref & & & & ref & & & \\
\hline & Rural area & -1.708 & -5.751 & 2.336 & 0.407 & 0.415 & -5.079 & 5.909 & 0.882 \\
\hline \multirow[t]{4}{*}{ Education } & Primary & ref & & & & ref & & & \\
\hline & Vocational & 3.935 & -1.936 & 9.806 & 0.188 & 10.716 & 2.738 & 18.693 & 0.009 \\
\hline & Secondary & 4.911 & -1.222 & 11.044 & 0.116 & 10.399 & 2.066 & 18.732 & 0.015 \\
\hline & Tertiary & 9.241 & 1.873 & 16.61 & 0.014 & 16.554 & 6.543 & 26.565 & 0.001 \\
\hline \multirow[t]{4}{*}{ Source of income } & Employed & ref & & & & ref & & & \\
\hline & Unemployed & 3.702 & -2.4 & 9.803 & 0.233 & 11.865 & 3.575 & 20.155 & 0.005 \\
\hline & Retired & 2.039 & -3.892 & 7.97 & 0.499 & 4.681 & -3.378 & 12.74 & 0.254 \\
\hline & $\begin{array}{l}\text { Retired on ill- } \\
\text { health grounds }\end{array}$ & -1.836 & -8.133 & 4.462 & 0.567 & -8.844 & -17.401 & -0.288 & 0.043 \\
\hline \multirow[t]{3}{*}{ Duration of diabetes } & Up to 5 years & ref & & & & ref & & & \\
\hline & $5-10$ years & -0.653 & -5.301 & 3.994 & 0.782 & -0.727 & -7.042 & 5.587 & 0.821 \\
\hline & Over 10 years & 1.017 & -3.581 & 5.615 & 0.664 & 3.31 & -2.937 & 9.557 & 0.298 \\
\hline \multicolumn{10}{|l|}{ (b) } \\
\hline & ACDS [points] & 1.59 & 1.073 & 2.106 & $<0.001$ & 1.834 & 1.401 & 2.268 & $<0.001$ \\
\hline \multirow[t]{2}{*}{ Sex } & Female & ref & & & & ref & & & \\
\hline & Male & 1.287 & -3.493 & 6.068 & 0.597 & -0.47 & -4.481 & 3.541 & 0.818 \\
\hline \multirow[t]{2}{*}{ Age } & Up to 60 years & ref & & & & ref & & & \\
\hline & Over 60 years & -1.883 & -9.179 & 5.414 & 0.612 & -2.944 & -9.066 & 3.179 & 0.345 \\
\hline \multirow[t]{3}{*}{ Place of residence } & Urban area & ref & & & & ref & & & \\
\hline & Rural area & 4.846 & -0.541 & 10.232 & 0.078 & 0.685 & -3.835 & 5.204 & 0.766 \\
\hline & Primary & ref & & & & ref & & & \\
\hline \multirow[t]{3}{*}{ Education } & Vocational & 4.439 & -3.383 & 12.26 & 0.265 & 7.64 & 1.077 & 14.202 & 0.023 \\
\hline & Secondary & 11.02 & 2.85 & 19.19 & 0.008 & 9.962 & 3.107 & 16.817 & 0.005 \\
\hline & Tertiary & 14.347 & 4.532 & 24.163 & 0.004 & 16.901 & 8.665 & 25.136 & $<0.001$ \\
\hline \multirow[t]{4}{*}{ Source of income } & Employed & ref & & & & ref & & & \\
\hline & Unemployed & 4.758 & -3.37 & 12.886 & 0.25 & 7.902 & 1.082 & 14.721 & 0.023 \\
\hline & Retired & 2.973 & -4.929 & 10.874 & 0.46 & 5.827 & -0.802 & 12.457 & 0.085 \\
\hline & $\begin{array}{l}\text { Retired on ill- } \\
\text { health grounds }\end{array}$ & -5.659 & -14.048 & 2.73 & 0.185 & -4.833 & -11.872 & 2.206 & 0.178 \\
\hline \multirow[t]{3}{*}{ Duration of diabetes } & Up to 5 years & ref & & & & ref & & & \\
\hline & $5-10$ years & 4.031 & -2.159 & 10.222 & 0.201 & 1.395 & -3.8 & 6.589 & 0.598 \\
\hline & Over 10 years & 0.723 & -5.402 & 6.848 & 0.817 & 2.432 & -2.708 & 7.571 & 0.353 \\
\hline
\end{tabular}

SCODI Self-Care of Diabetes Index, ACDS Adherence in Chronic Diseases Scale, $N$ number of patients, $R$ regression parameter, $C I$ confidence interval, $p$ level of statistical significance

status showed a lower level of adherence. In other study by García et al. [26] on the management of DM and adherence to treatment showed that self-efficacy was a significant predictor of later adherence to treatment of DM. Based on a cross-sectional study conducted among 419 patients with type 2 diabetes, Bonger et al. [10], showed a lack of adherence to dietary recommendations in more than $75 \%$ of the respondents. As many as 83.5 patients did not adhere to selfmonitoring of blood glucose level and almost $20 \%$ were not taking the prescribed medications. 
The other study by Shrivastava et al. [5] stressed the importance of both patient-related factors, such as adherence to recommendations, proactive attitude, convictions, knowledge of diabetes, financial resources, co-morbidities and social support, and factors relating to medical teams, such as effective communication, knowledge and treatment planning skills, as factors which determine the success of treatment.

Our study demonstrates the importance of sociodemographic factors in managing type $2 \mathrm{DM}$. We found that female patients, patients with tertiary education and unemployed patients showed a higher level of self-care behaviours. Bonger et al. [10] investigated self-care in 419 patients with type $2 \mathrm{DM}$ and found that $75.9 \%$ of the patients failed to adhere to dietary recommendations, while $83.5 \%$ of the patient sample did not monitor their blood glucose levels. Moreover, the study of Bonger et al. [10] showed that unemployed patients, patients with tertiary education and younger patients were more likely to adhere to treatment regimens. Tekalegn et al. [27] list the duration of the disease and the type of treatment used among the determinants influencing adherence to the therapeutic recommendations. In a group of over 400 patients, the predictors of worse glycemic control were longer disease duration and insulin therapy.

Recognising the multidimensional nature of the problem, systematic and multifaceted approach must be taken to improve the level of self-care in patients with DM to improve adherence and the effectiveness of treatment. Achieving high levels of therapeutic adherence in diabetic patients should be a key objective for health-care workers. The available literature also suggests, that new and innovative approaches need to be encouraged, clinically tested, and then implemented.

Accoridng to the implications for clinical practice, it is recommended that a routine assessment of the level of self-care, including adherence, be carried out in patients diagnosed with type 2 DM. Early identification of knowledge deficits about self-care is of vital importance for the provision of health education tailored to patients' needs. Improved adherence to treatment in patients with type 2 DM may help prevent or delay complications of the condition. The study clearly indicates the importance and role of self-care in achieving a satisfactory level of the adherence with the therapeutic recommendations. There is a need to pay attention to the phenomenon of self-care and self-management in the group of patients with type 2 diabetes. The assessment of the quality of care in the discussed population seems to be important. Such actions will allow monitoring of patients' self-management [28]. The use of health education is an important tool to support and promoting behaviours that contribute to improving self-care among type 2 diabetes patients [8]. Health education should therefore be an integral part of clinical and outpatient practice to support patients with type 2 diabetes.

\section{Conclusions}

The self-care management behaviours have a significant impact on adherence to treatment in patients with type 2 DM. The higher the level of self-efficacy in each of the areas of functioning, the higher adherence. It was found that female patients, patients with tertiary education and unemployed patients showed a higher level of type 2 DM self-care.

\section{Limitations of the study}

The results of the study clearly indicate the importance of self-care for adherence in the group of patients with type 2 diabetes. The limitation of the study was the lack of analysis of clinical parameters (e.g. blood glucose and therapeutic haemoglobin (HbA1c) levels) indicating adherence to therapeutic recommendations and their correlation with the domains of the self-care questionnaire. It seems necessary to conduct further research using health education and to assess its impact on adherence and selfare.

Acknowledgments There were no other contributors to the article than the Authors as well as there was no writing assistance required. The certificated English language services were provided by an academic highly qualified native speaker.

Author contributions SK and IU conceived of the study concept and developed the study design. $\mathrm{KL}$ and AC were responsible for data collection and analysis. SK, KL and AC drafted the manuscript. SK and IU were responsible for the data interpretation. IU and DA revised the final draft. All authors approved the final version to be published.

Funding This study was funded by the Ministry of Science and Higher Education of Poland as a statutory grant of the Wroclaw Medical University for maintaining research potential (no. SUB.E020.19.003).

Data availability The datasets generated during and/or analysed during the current study are available from the corresponding author on reasonable request.

\section{Compliance with ethical standards}

Conflict of interest The authors declare that they have no conflict of interest.

Ethics approval The protocol performed in this study was in accordance with the ethical standards of the institutional and/or national research committee and with the 1964 Helsinki Declaration and its later amendments or comparable ethical standards. The study protocol was approved by the Wroclaw Medical University Bioethics Committee (approval no. KB-622/2018).

Consent to participate All patients provided informed consent prior to their inclusion in the study. The study was carried out in accordance with the tenets of the Declaration of Helsinki and Good Clinical Practice guidelines. 
Consent to publish Not applicable.

Human and animal rights The protocol performed in this study was in accordance with the ethical standards of the institutional and/or national research committee and with the 1964 Helsinki Declaration and its later amendments or comparable ethical standards. The study protocol was approved by the Wroclaw Medical University Bioethics Committee (approval no. KB-622/2018).

Informed consent All patients provided informed consent, and were informed that they could withdraw from the study at any time. The study was carried out in accordance with the tenets of the Declaration of Helsinki and Good Clinical Practice guidelines.

Open Access This article is licensed under a Creative Commons Attribution 4.0 International License, which permits use, sharing, adaptation, distribution and reproduction in any medium or format, as long as you give appropriate credit to the original author(s) and the source, provide a link to the Creative Commons licence, and indicate if changes were made. The images or other third party material in this article are included in the article's Creative Commons licence, unless indicated otherwise in a credit line to the material. If material is not included in the article's Creative Commons licence and your intended use is not permitted by statutory regulation or exceeds the permitted use, you will need to obtain permission directly from the copyright holder. To view a copy of this licence, visit http://creativecommons.org/licenses/by/4.0/.

\section{References}

1. World health organization (2016) global report on diabetes. World health organization, Geneva, Switzerland. https://apps.who.int/iris/ bitstream/handle/10665/204871/9789241565257_eng.pdf;jsessionid $=967551616 \mathrm{BC} 22 \mathrm{BAC} 22 \mathrm{BFE} 0 \mathrm{~F} 8 \mathrm{~F} 50 \mathrm{~B} 556 \mathrm{~A}$ ? sequence $=1$

2. Zheng Y, Ley SH, Hu FB (2018) Global aetiology and epidemiology of type 2 diabetes mellitus and its complications. Nat Rev Endocrinol 14:88-98. https://doi.org/10.1038/nrendo.2017.151

3. Cho NH, Shaw JE, Karuranga $\mathrm{S}$ et al (2018) IDF diabetes atlas: global estimates of diabetes prevalence for 2017 and projections for 2045. Diabetes Res Clin Pract 138:271-281. https://doi. org/10.1016/j.diabres.2018.02.023

4. Inzucchi SE, Bergenstal RM, Buse JB et al (2012) Management of hyperglycemia in type 2 diabetes: a patient-centered approach. Diabetes Care 35:1364-1379. https://doi.org/10.2337/dc12-0413

5. Shrivastava SR, Shrivastava PS, Ramasamy J (2013) Role of selfcare in management of diabetes mellitus. J Diabetes Metab Disord 12:14. https://doi.org/10.1186/2251-6581-12-14

6. American Diabetes Association (2014) Standards of medical care in diabetes-2014. Diabetes Care 37(Suppl 1):14-80. https://doi. org/10.2337/dc14-S014

7. Afridi MAR, Khan MN (2003) Role of health education in the management of diabetes mellitus. J Coll Physicians Surg Pak 13:558561. https://doi.org/10.2003/JCPSP.558561

8. Shabibi P, Zavareh MSA, Sayehmiri K, et al (2017) Effect of educational intervention based on the health belief model on promoting self-care behaviours of type- 2 diabetes patients. Electron Physician 9:5960-5968. https://doi.org/10.19082/5960

9. Zulman DM, Rosland A-M, Choi H et al (2012) The influence of diabetes psychosocial attributes and self-management practices on change in diabetes status. Patient Educ Couns 87:74-80. https://doi. org/10.1016/j.pec.2011.07.013

10. Bonger Z, Shiferaw S, Tariku EZ (2018) Adherence to diabetic selfcare practices and its associated factors among patients with type 2 diabetes in Addis Ababa, Ethiopia. Patient Prefer Adher 12:963970. https://doi.org/10.2147/PPA.S156043
11. Miller TA, DiMatteo MR (2013) Importance of family/social support and impact on adherence to diabetic therapy. Diabetes Metab Syndr Obes 6:421-426. https://doi.org/10.2147/DMSO.S36368

12. Costa E, Giardini A, Savin M et al (2015) Interventional tools to improve medication adherence: review of literature. Patient Prefer Adher 9:1303-1314. https://doi.org/10.2147/PPA.S87551

13. Adu MD, Malabu UH, Malau-Aduli AEO, Malau-Aduli BS (2019) Enablers and barriers to effective diabetes self-management: a multinational investigation. PLoS ONE. https://doi.org/10.1371/journ al.pone.0217771

14. Ausili D, Barbaranelli C, Rossi E et al (2017) Development and psychometric testing of a theory-based tool to measure self-care in diabetes patients: the self-care of diabetes inventory. BMC Endocr Disord 17:66. https://doi.org/10.1186/s12902-017-0218-y

15. Uchmanowicz I, Krzemińska S, Ausili D et al (2020) Polish Adaptation of the self-care of diabetes inventory (SCODI). Patient Prefer Adher 14:1341-1350. https://doi.org/10.2147/PPA.S253444

16. Kubica A, Kosobucka A, Michalski P et al (2017) Adherence in chronic disease scale-a new instrument to evaluate adherence to the treatment plan. Folia Cardiol 12:19-26. https://doi.org/10.5603/ FC.a2016.0105

17. R Core Team (2019) R: a language and environment for statistical computing. R Foundation for Statistical Computing, Vienna, Austria

18. Song Y, Nam S, Park S et al (2017) The impact of social support on self-care of patients with diabetes: What is the effect of diabetes type? systematic review and meta-analysis. Diabetes Educ 43:396412. https://doi.org/10.1177/0145721717712457

19. Alhaik S, Anshasi HA, Alkhawaldeh J et al (2019) An assessment of self-care knowledge among patients with diabetes mellitus. Diabetes Metab Syndr 13:390-394. https://doi.org/10.1016/j.dsx.2018.10.010

20. Araszkiewicz A, Bandurska-Stankiewicz E, Budzyński A et al (2019) 2019 guidelines on the management of diabetic patients. A position of diabetes Poland. Clin Diabetol 8:1-95. https://doi. org/10.5603/DK.2019.0001

21. Powers MA, Bardsley J, Cypress M et al (2016) Diabetes selfmanagement education and support in type 2 diabetes: a joint position statement of the american diabetes association, the american association of diabetes educators, and the academy of nutrition and dietetics. Clin Diabetes 34:70-80. https://doi.org/10.2337/diacl in.34.2.70

22. Brunisholz KD, Briot $P$, Hamilton $S$ et al (2014) Diabetes self-management education improves quality of care and clinical outcomes determined by a diabetes bundle measure. J Multidiscip Healthc 7:533-542. https://doi.org/10.2147/JMDH.S69000

23. Mikhael EM, Hassali MA, Hussain SA (2020) Effectiveness of diabetes self-management educational programs for type 2 diabetes mellitus patients in middle east countries: a systematic review. Diabetes Metab Syndr Obes 13:117-138. https://doi.org/10.2147/ DMSO.S232958

24. Gonzalez-Zacarias AA, Mavarez-Martinez A, Arias-Morales CE et al (2016) Impact of demographic, socioeconomic, and psychological factors on glycemic self-management in adults with type 2 diabetes mellitus. Front Public Health 4:195. https://doi.org/10.3389/ fpubh.2016.00195

25. Polonsky WH, Henry RR (2016) Poor medication adherence in type 2 diabetes: recognizing the scope of the problem and its key contributors. Patient Prefer Adher 10:1299-1307. https://doi.org/10.2147/ PPA.S106821

26. Brown SA, García AA, Brown A et al (2016) Biobehavioral determinants of glycemic control in type 2 diabetes: a systematic review and meta-analysis. Patient Educ Couns 99:1558-1567. https://doi. org/10.1016/j.pec.2016.03.020

27. Tekalegn Y, Addissie A, Kebede T, Ayele W (2018) Magnitude of glycemic control and its associated factors among patients with type 2 diabetes at tikur anbessa specialized hospital, Addis Ababa 
Ethiopia. PLoS ONE 13:e0193442. https://doi.org/10.1371/journ al.pone.0193442

28. Ansari RM, Dixon JB, Browning CJ (2014) Self-management of type 2 diabetes in middle-aged population of Pakistan and Saudi Arabia. Open J Prev Med 4:396-407. https://doi.org/10.4236/ ojpm.2014.46047
Publisher's Note Springer Nature remains neutral with regard to jurisdictional claims in published maps and institutional affiliations. 